\title{
Studies on Transplantation*
}

\section{T. E. STARZL, T. L. MARCHIORO, Y. IWASAKI, N. KASHIWAGI}

Department of Surgery, University of Colorado School of Medicine

and the Denver Veterans Administration tiospital,

Denver, Colorado

There is now ample evidence that homotransplantation of whole vital organs will be a useful and increasingly important method of therapy in the coming years. The attitude of gloom with which both physicians and basic scientists viewed this possibility only recently has been replaced by a sometimes unbridled optimism.

The principal reasons for this change have been the demonstrations that many uremic patients can be materially benefited by homotransplantation of the kidney, that rejection is an inherently and often easily reversible phenomenon, that the need for stringent immunosuppressive therapy tends to decrease with the passage of time, and that the patient with a really good result can live an essentially normal life.

\section{PAST EFFORTS}

The facts mentioned above became evident soon after the now widely used combination of immunosuppressive drugs was evolved. Using most importantly azathioprine and prednisone, and in some cases actinomycin $\mathrm{C}$ and local homograft irradiation, 64 patients were treated in Denver from November 1962 to March 1964, employing homografts from volunteer living donors. Today, now $2 \frac{1}{2}$ to almost 4 years later, exactly half of these recipients are still alive by virtue of their still chronically functioning kidneys. The principal mortality was during the first few postoperative months (Fig. 1), after which the chance of continuing survival was relatively good.

*Aided by United States Public Health Service grants AM06283, HE 07735, AM 07772, AI 04152, FR 00051 and FR 00064. 
There was a striking difference in the results, depending upon the source of the homograft. Forty-six of the foregoing recipients received kidneys from consanguineous donors; $29(63 \%)$ are still alive. In contrast, only 3 of 18 patients who received nonrelated kidneys survive to date (Fig. 2).

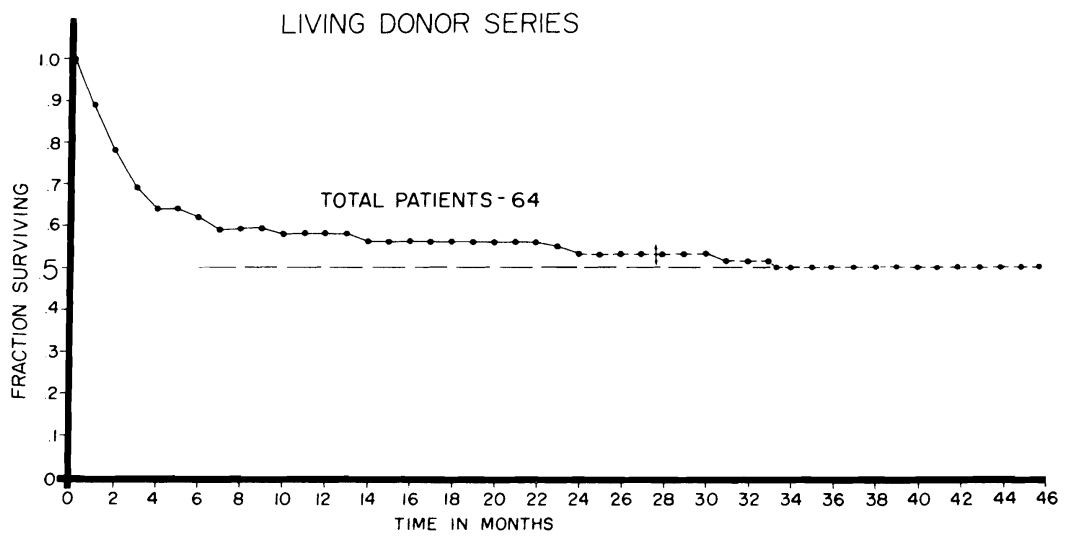

FIG. 1. Life survival curve of the first sixty-four patients who received renal homotransplants from November 1962 to March 1964. All donors were living volunteers. The recipients now have been followed for $21 / 2$ to almost 4 years. Exactly half of the patients were still alive on September 26, 1966.

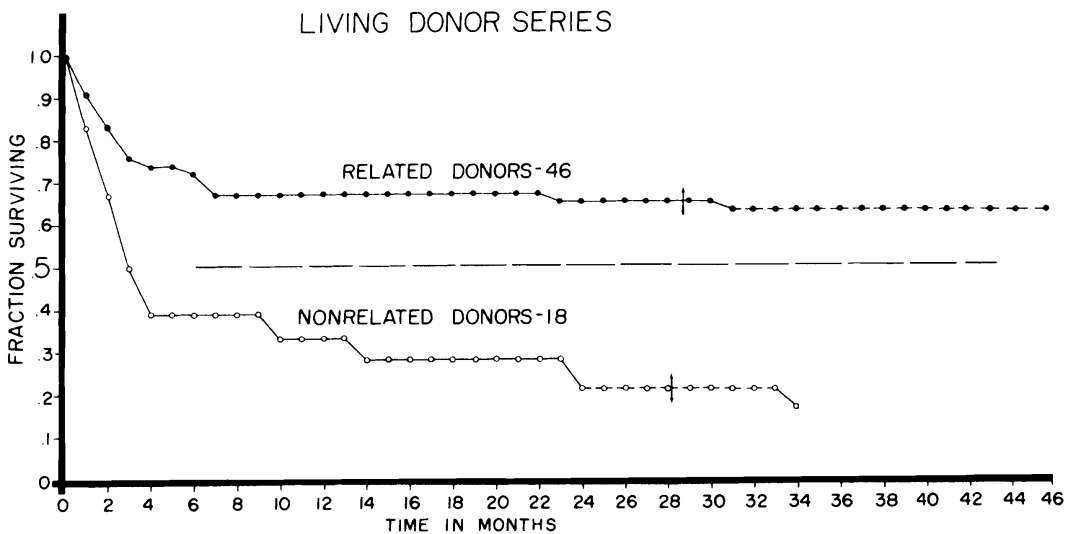

FIG. 2. A breakdown of the results shown in Fig. 1 according to the source of the homograft. Note that $63 \%$ of the patients who had consanguineous donors are still alive compared to only 3 of 18 who received nonrelated kidneys.

This experience was accumulated at a time when the clinical problems which were to be encountered were either understood poorly or not at all. 
Nevertheless, the substantial salvage rate obtained has proved in principle the feasibility of the undertaking. Furthermore, these, as well as similar results from other institutions (Hamburger et al., 1965; Hume et al., 1966; Murray et al., 1965; Straffon et al., 1966), have defined areas into which intensive effort has already been invested.

\section{CAUSES OF FAILURE}

The vast majority of deaths after renal homotransplantation are more or less directly caused by the immunosuppressive agents used whether this occurs early, at an intermediate time, or late. In turn, the toxicity of the various agents is related to the difficulty with which rejection is controlled.

A pertinent example is shown in Fig. 3. The patient, who received a

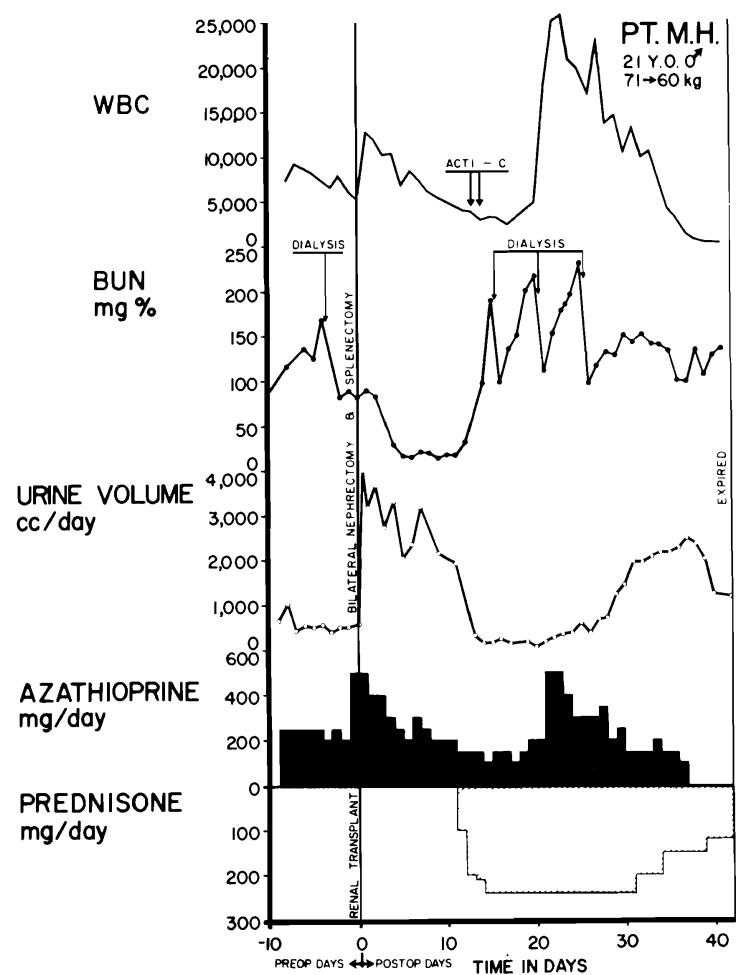

FIG. 3. Typical unsuccessfully treated case. The donor and recipient were brothers, both of A+ blood type. A violent rejection crisis followed good early function, and anuria developed which lasted 2 weeks. Although the rejection was reversed and a secondary diuresis began, the patient died from drug toxicity, leukopenia, and septicemia. Acti C, each arrow is $200 \mu \mathrm{g}$ intravenous actinomycin C. (By permission of Surgery 56, 296, 1964.) 
kidney from his brother, had good renal function for more than week. Severe rejection then ensued, causing temporary renal shutdown, but with maximum immunosuppression, urinary output resumed. In spite of this temporary improvement, the patient died shortly afterward of fungal and bacterial sepsis which were primarily the consequence of bone marrow depression. The responsible drug was azathioprine. It has since been appreciated that the dose of this agent must be reduced during periods of depressed urine excretion inasmuch as the drug has an important renal pathway of detoxification. Dose control is extremely difficult under these circumstances.

With increased experience, such deaths from acute bone marrow depression have become rare, and it seems probable that azathioprine is the safest of the immunosuppressive drugs being used for chronic therapy. When azathioprine can be used as the sole therapeutic agent, infectious complications or other morbidity are negligible. Unfortunately, this is frequently not possible. It is often found that continued homograft function can be maintained only with the additional use of substantial quantities of prednisone. It is the need for high dose steroid therapy which most severely limits the life expectancy of any individual patient.

The tragic consequences which may ensue during steroid therapy, either early or late, are illustrated in Fig. 4. This patient, who had a severe late rejection almost a year after operation, needed large doses of prednisone to prevent further deterioration of homograft function. As a consequence, he developed typical cosmetic deformities, bone demineralization, a duodenal ulcer, pancreatitis, and parenchymal liver disease. Until the time of death, his renal function was adequate to sustain life in a normal person. At autopsy, he had pneumonitis and infestation of several organ systems with cytomegalic inclusion virus. Such patients often become infected with fungi or bacteria which ordinarily have a low grade pathogenicity.

\section{HISTOCOMPATIBILITY MATCHING}

Since toxicity from immunosuppression accounts for a heavy mortality, usually because large doses are mandatory, intensive efforts are being made in several centers to sharpen the criteria by which a donor is decided upon for any given recipient. With proper selection, it would be expected that the need for potentially lethal immunosuppressive measures could be at least partially eliminated. This approach has been evaluated in collaboration with Dr. Paul Terasaki of Los Angeles, whose method of lymphocyte analysis appears to measure something which is at least related to histocompatibility antigens (Porter et al., 1966; Terasaki et al., 1966). 


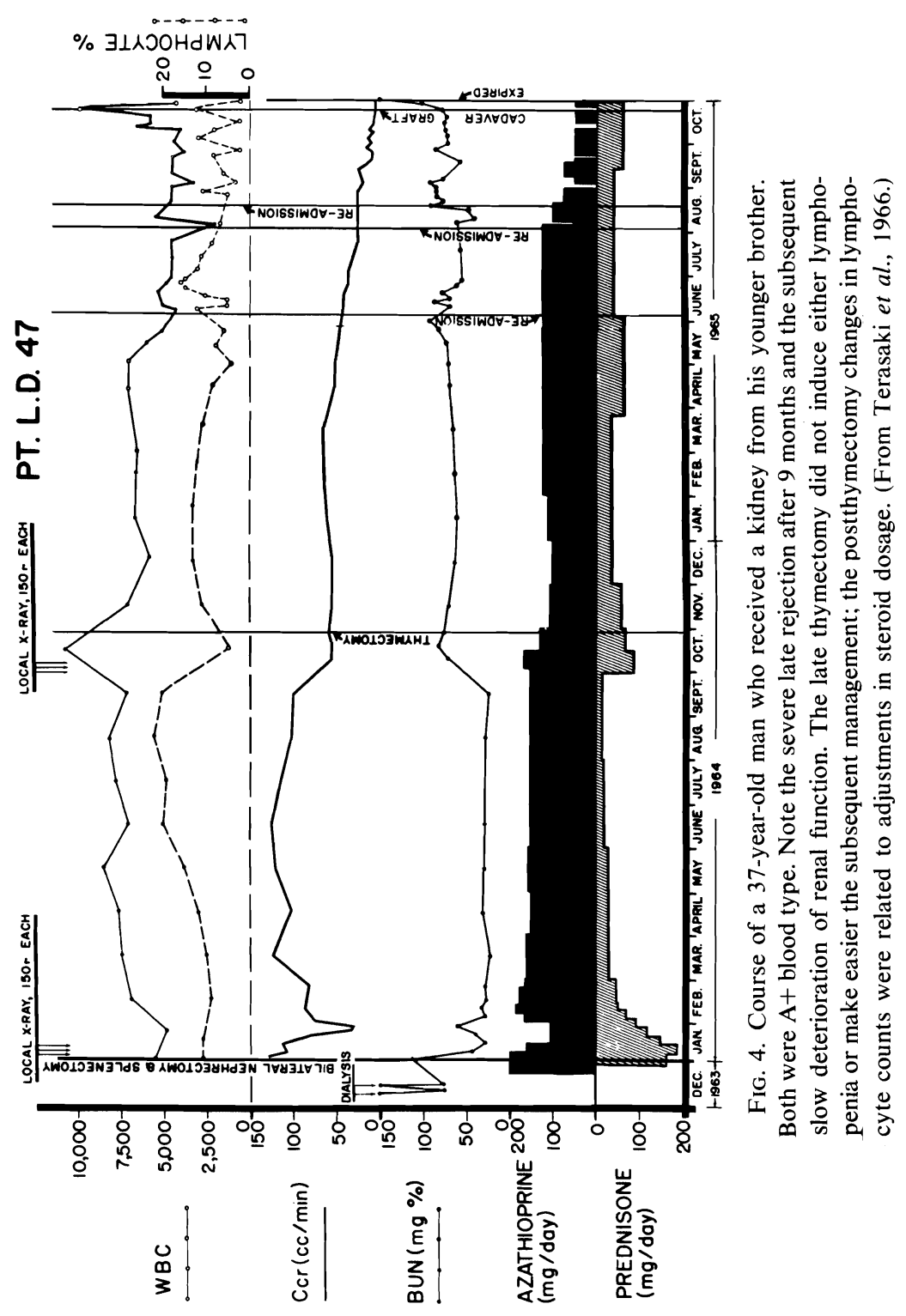


The fate of the first 26 patients for whom donors were chosen by Terasaki on the basis of the best available antigen match is shown in Fig. 5. The transplantations were between blood relatives in half the cases, and between nonrelated pairs in the other half. After a follow-up of 12 to 22 months, the results were exactly the same in the two subgroups. The upgrading was expected in the unrelated subgroup if the test had any discriminatory capability. Failure to increase survival in recipients of related kidneys was a disappointment. The drug toxicity described earlier was still the chief cause of death in both groups. It became clear that the high mortality could not be eliminated even with effective typing procedures without the evolution of better immunosuppressive treatment.

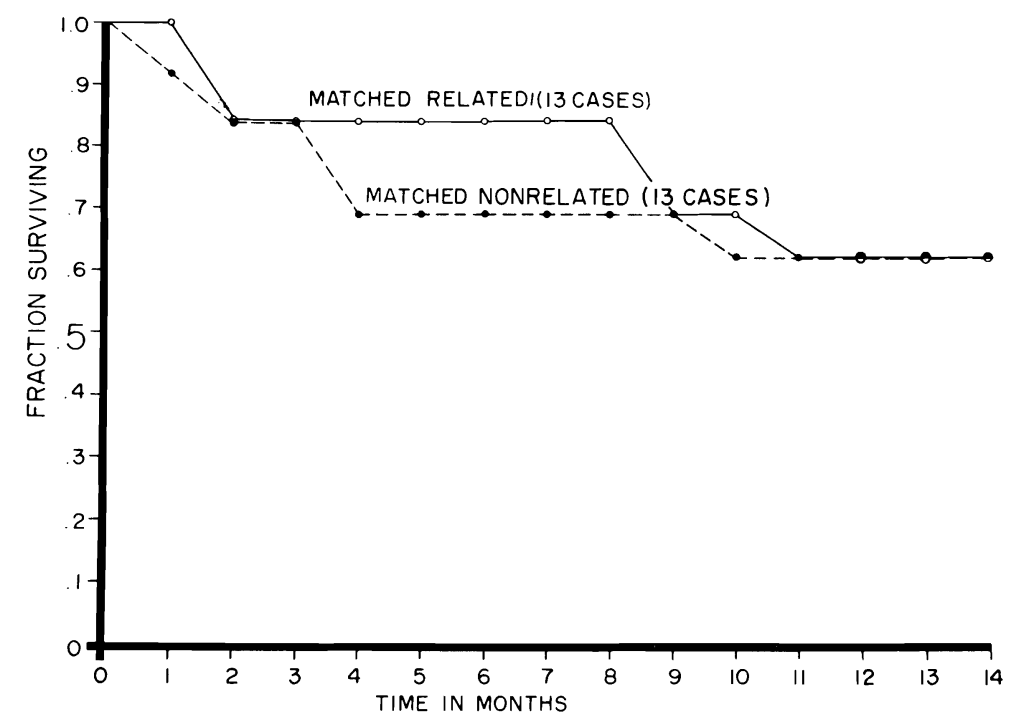

FIG. 5. Results of renal transplantation in a more recent series of patients whose donors were selected by Terasaki's antigen matching method. Note that survival after 13-22 months was exactly the same in both the related and nonrelated cases. Compare these results with those shown in Fig. 2.

\section{IMPROVED IMMUNOSUPPRESSION}

The most promising new treatment we have used is antilymphoid serum, raised in a heterologous host by immunization with lymphoid tissue from the species to be eventually treated. Such a product was described by Metchnikoff in 1899 and by numerous others in the ensuing 60 years. Its use to potentiate homograft survival was first suggested by Woodruff (1960; Woodruff and Anderson, 1964) and Waksman et al. 
(1961). The value of antilymphoid serum for this purpose has been established in a number of subsequent studies (Gray et al., 1964; Jeejeebhoy, 1965; Levey and Medawar, 1966a,b; Monaco et al., 1965, 1966; Nagaya and Sieker, 1965).

In our laboratories, antilymphoid derivatives have been developed for use in both dogs and man (Iwasaki et al., 1967). Horses were subcutaneously immunized with lymphocytes from the spleen, lymph nodes, or thymus of the appropriate species. The equine leukoagglutinin and lymphoagglutinin titers rose to as high as $1: 16,000$ (Fig. 6). The horse serum which was toxic in its raw state was absorbed with pooled red cells and serum of the species which provided the immunizing tissue.

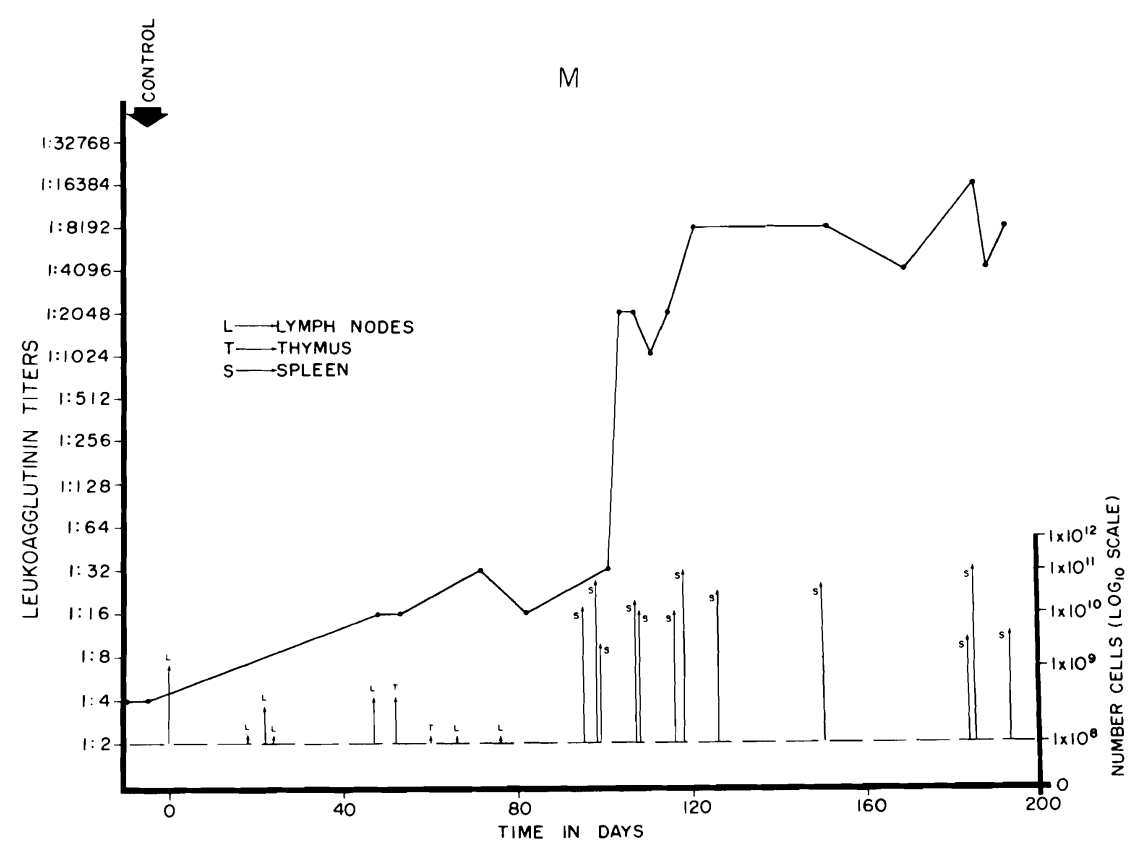

FIG. 6. Effect of immunizing dose upon the leukoagglutinin titer of a horse inoculated with cadaveric human lymphoid tissue. Note that the rise in titer was very modest during the first 3 months, during which time small doses of cells were used. When the quantity of antigen was increased by the use of spleen cells, abrupt increases in titer were observed within a few days. (From Iwasaki et al., 1967.)

Next, studies were conducted to localize the desired antibody (Iwasaki et al., 1967). By separation with the DEAE cellulose column, and by determination of the leukoagglutinating titers and electrophoretic characteristics of the various eluates, it was found that the antiwhite cell antibody was chiefly in $\gamma$-globulin with lesser activity in $\beta$-globulin (Fig. 
7). These constituents could easily be removed by double or quadruple precipitation with 0.4 saturation ammonium sulfate (Fig. 8).

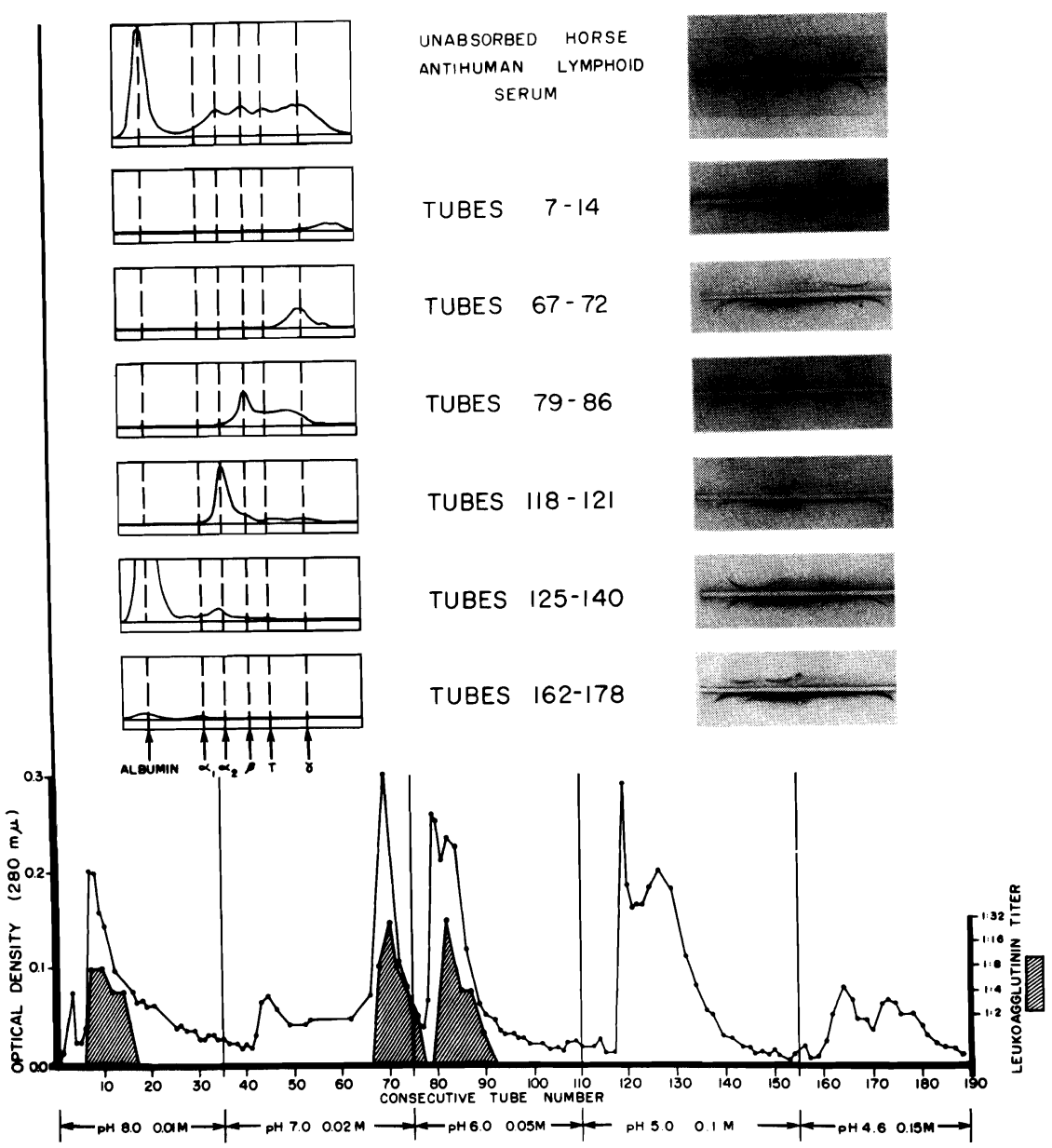

FIG. 7. Studies of the leukoagglutinin-containing fractions in antihuman-lymphoid serum employing column chromatography, electrophoresis, and immunoelectrophoresis. The various eluates from the DEAE cellulose column were analyzed spectrophotometrically for protein content (expressed as optical density), and the presence or absence of leukoagglutinins determined for each collection tube. The electrophoresis and immunoelectrophoresis permitted relatively complete classification of the active immunoglobulins. (From Iwasaki et al., 1967.)

Ammonium sulfate-precipated antidog-lymphoid globulin has been extensively studied in the laboratory (Iwasaki et al., 1967; Starzl et al., 1967). When administered subcutaneously as the sole therapy, it pro- 
ELECTROPHORESIS

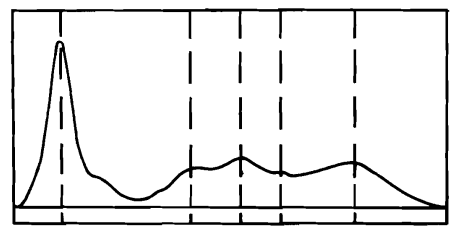

ABSORPTION AND PRECIPITATION WITH 0.4 SAT. $\left(\mathrm{NH}_{4}\right)_{2} \mathrm{SO}_{4}$

$\downarrow$
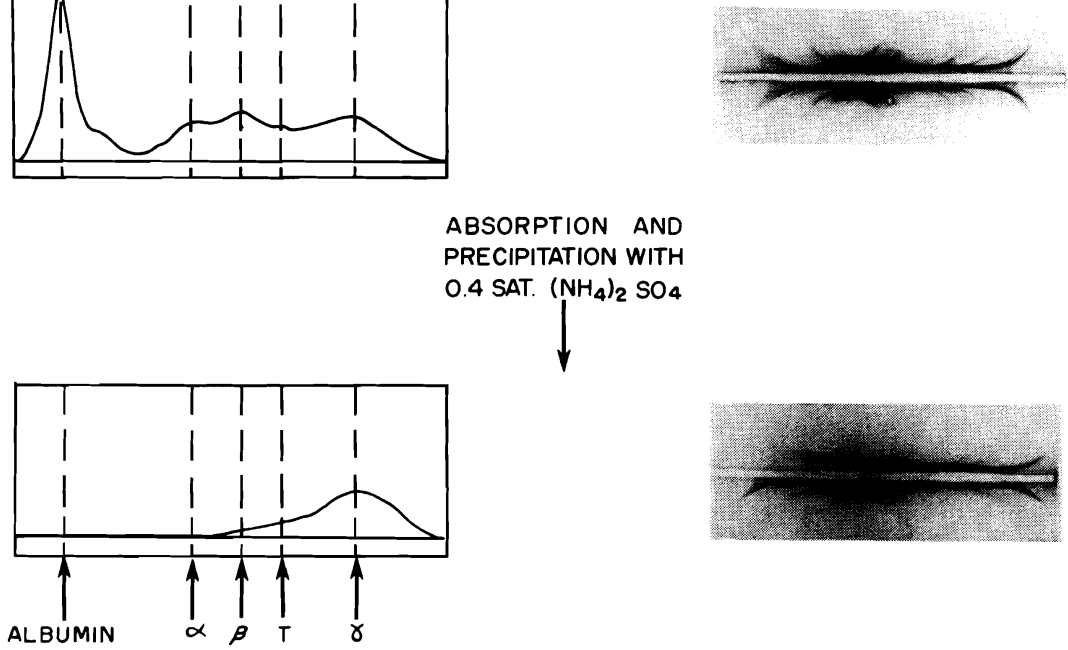

FIG. 8. Electrophoresis and immunoelectrophoresis of absorbed antihuman-lymphoid serum and the protein obtained from it by four precipitations with 0.4 saturated ammonium sulfate, four dialyses and lyophilization. The final product, which was used clinically, consists almost entirely of $\gamma \mathrm{G}$ globulin. (From Iwasaki et al., 1967.)

longed the survival of either kidney or liver homografts (Fig. 9) only slightly less than azathioprine (Starzl et al., 1967). There was little overt toxicity although a substantial fraction of either control or transplanted kidneys developed electron-dense bodies in the glomeruli (Iwasaki et al., 1967) which did not cause a demonstrable reduction in function. The studies indicated that the immune globulin was relatively safe, at least for short-term therapy, but that it was not potent enough to warrant use as the sole method of immunosuppression.

For clinical trial, antihuman-lymphoid globulin was therefore combined with the older standard drugs, azathioprine and prednisone (Starzl et al., 1967). As in the past, a conscious effort was made to use as little prednisone as possible. Eight patients were treated from 9 weeks to 3 months ago. Their renal function, drug doses, and peripheral hematologic patterns during the first 9 postoperative weeks were then compared to those of three groups of previously treated recipients in which the homograft sources had been comparable.

The results are shown in Fig. 10. During the period in question, only $46 \%$ as much prednisone was required as for any of the preceding series. The renal function was comparable. The peripheral lymphocytes were 


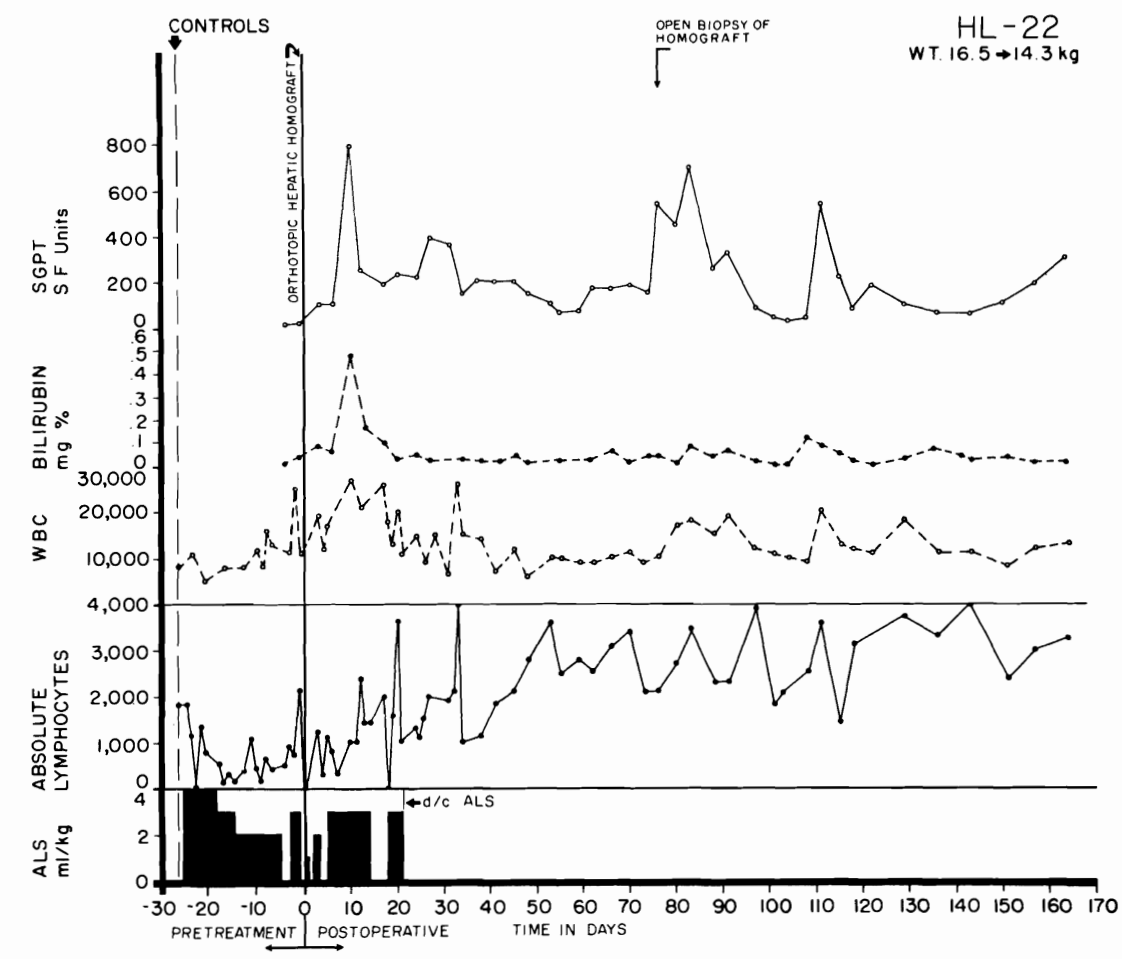

FIG. 9. Course of a dog after orthotopic liver transplantation. The donor and recipient were nonrelated mongrel animals. The only immunosuppression used was horse antilymphoid serum given intraperitoneally. This treatment was stopped 3 weeks postoperatively and the animal has lived for more than 5 subsequent months during all of which time the peripheral lymphocyte count has been high. (From Starzl et al., 1967.)

little changed, a finding which is consonant with the brilliant studies of Levey and Medawar $(1966 a, b)$ who have shown that the immunosuppressive effect is not dependent upon either lymphoid depletion or lymphopenia. All eight patients are clinically well.

The aforementioned studies are purely investigational. Whether longterm toxicity, including foreign protein injury to the homograft itself, will occur, can only be determined with longer follow-up. These data do indicate, however, that the possibility of better immunosuppression is not just a mirage.

\section{OTHER ORGANS}

Clinically, only the kidney has been transplanted successfully to date. It seems inevitable that other organs will follow. In our laboratories, dogs 


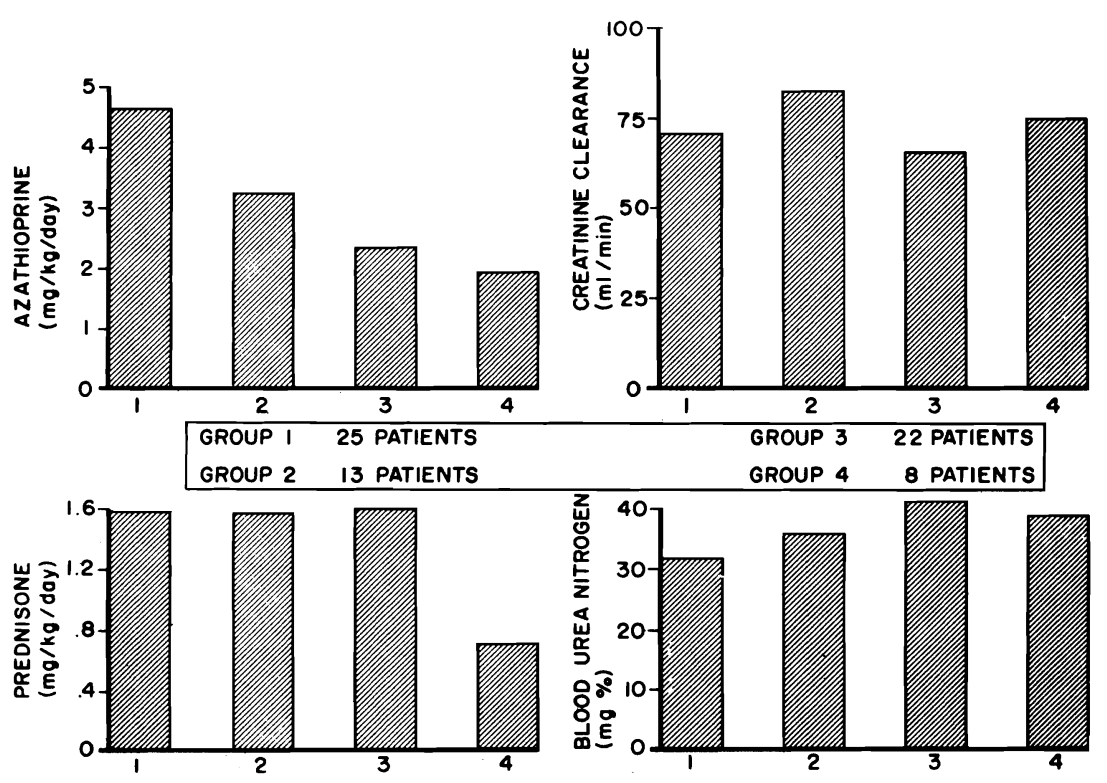

Fig. 10. Variations in immunosuppression and renal function during the first 63 postoperative days in four successive groups of patients who received kidneys from blood relatives.. Since the blood urea nitrogen and creatinine clearance were not determined each day, these were compiled on a weekly basis. Those in series 4 received adjuvant therapy with antilymphoid globulin. Note the drastic reduction in average prednisone dose which was achieved in these patients without significant loss of renal function. (From Starzl et al., 1967.)

are alive as long as $2 \frac{1}{2}$ years after complete hepatectomy and replacement with a homograft from a nonrelated mongrel donor (Starzl et al., 1965). In our experience, this kind of chronic survival can be achieved more often with hepatic than with renal homografts. Other workers have had long-term success with cardiac and pulmonary homotransplantation.

\section{SUMMARY}

Results with clinical renal homotransplantation have been reviewed. The patterns of mortality have been cited as these relate to research in histocompatibility typing and in the search for better immunosuppressive regimens.

\section{References}

Gray, J. G., Monaco, A. P., and Russell, P. S. (1964). Surg. Forum 15, 142.

Hamburger, J., Crosnier, J., Dormont, J., Reveillard, R.-J., Hors, J.-H., and Alsina, J. (1965). Presse Med. 73, 2793. 
Hume, D. M., Lee, H. M., Williams, G. M., Wolf, J. S., Prout, G. R., Slapak, M., Kauffman, H. M., Jr., and Cleveland, R. J. (1966). Ann. Surg. 164, 352.

Iwasaki, Y., Porter, K. A., Amend, J. R., Marchioro, T. L., Zuhlke, V., and Starzl, T. E. (1967). Surg. Gynecol. Obstet. 124, 1.

Jeejeebhoy, H. F. (1965). Immunology 9, 417.

Levey, R. H., and Medawar, P. B. (1966a). Ann. N.Y.Acad.Sci. 129, 164.

Levey, R. H., and Medawar, P. B. (1966b). Proc. Natl.Acad. Sci. U.S. 56, 1130.

Metchnikoff, E. (1899). Ann. Inst. Pasteur 13, 737.

Monaco, A. P., Wood, M. L., and Russell, P. S. (1965). Surg. Forum 16, 209.

Monaco, A. P., Wood, M. L., Gray, J. G., and Russell, P. S. (1966). J. Immunol. 96, 229.

Murray, J. E., Gleason, R., and Bartholomay, A. (1965). Transplantation 3, 684.

Nagaya, H., and Sieker, H. O. (1965). Science 150, 1181.

Porter, K. A., Rendall, J. M., Stolinski, C., Terasaki, P. I., Marchioro, T. L., and Starzl, T. E. (1966). Ann. N.Y.Acad.Sci. 129, 615.

Starzl, T. E. (1964). "Experience in Renal Transplantation." Saunders, Philadelphia, Pennsylvania.

Starzl, T. E., Marchioro, T. L., and Porter, K. A. (1966). In "Advances in Surgery" (C. Welch, ed.), pp. 295-370. Year Book Publ., Chicago, Illinois.

Starzl, T. E., Marchioro, T. L., Porter, K. A., Iwasaki, Y., and Cerilli, J. C. (1967). Surg. Gynecol. Obstet. 124, 301.

Straffon, R. A., Hewitt, C. B., Kiser, W. S., Stewart, B. H., Nakamoto, S., and Kolff, W. J. (1966). Surg. Gynecol. Obstet. 123, 483.

Terasaki, P. I., Porter, K. A., Marchioro, T. L., Mickey, M. R., Vredevoe, D. L., Faris, T. D., and Starzl, T. E. (1966). Ann. N.Y. Acad.Sci. 129, 500.

Waksman, B. H., Arbouys, S., and Arnason, B. G. (1961). J. Exptl. Med. 114, 997.

Woodruff, M. F. A. (1960). "The Transplantation of Tissues and Organs." Thomas, Springfield, Illinois.

Woodruff, M. F. A., and Anderson, N. F. (1964). Ann. N.Y.Acad. Sci. 120, 119.

\section{DISCUSSION}

DR. BEREnBAum: Have you any information on the susceptibility to infections of dogs or patients treated with antilymphocyte sera as compared with conventionally treated cases?

DR. STARZL: We do not have a precise quantitative evaluation in this respect as we do in patients with Imuran and prednisone. We have had no infections in patients treated with immune globulin and, in animals, infections have not been a prominent cause of failure. The patients are extraordinarily well off after treatment with antilymphoid globulin given in combination with azathioprine and small doses of steroids.

Dr. Amos: What is really known about the physiological adaptation to immunosuppressive drugs? The patients starting off on azathioprine are often in a very unstable position from the beginning. As you pointed out, in dogs one can stop drug treatment at a certain time after grafting, and an adaptation seems to occur between the transplant and the host. There may be also an adaptation of the host to the drug, and if we knew exactly what the individual drug requirements of a patient were, I think this might help in reducing initial mortality.

DR. STARZL: Yes, it would help very much, because there is a great individual difference. Some patients can tolerate only $0.5 \mathrm{mg} / \mathrm{kg}$ and some 4 or $5 \mathrm{mg} / \mathrm{kg}$.

DR. BACH: It was encouraging to see the survival of kidneys from unrelated donors matched by leukocyte typing as compared to the survival of those from related donors that 
you observed. I wonder how easy it was to find the unrelated donors who matched to the degree that they were matched.

Dr. STARzL: The results obtained earlier with randomly selected related donors were quite good and were not significantly improved by matching according to leukocyte typing. Very often, within the related group, there is not a large number of people from whom to select and therefore the quality of the match cannot be significantly improved by leukocyte typing. Also, within a family, the number of really bad matches is relatively small. In a nonrelated population, however, the chances of getting a bad match are exceedingly high. These may be the reasons why in nonrelated groups, but not in related groups, a significant improvement in the matching could be achieved following the criteria of leukocyte typing.

Dr. AMIEL: I would like to comment on the possibility of successfully selecting a donor to match the prospective recipient by phenotyping leukocyte antigens. Experiments in mice performed in our laboratory showed that, when bone marrow cells from more than one donor are transplanted into one recipient, those from the most compatible donor prevail and can be recovered some weeks after grafting. In a clinical case, four members of the family of a prospective recipient (father, mother, and two brothers of the mother) and two unrelated donors chosen from a list of about fifty volunteers on the basis of leukocyte typing (agglutination and cytotoxicity) served as bone marrow donors. Two months later, it was found that the successful graft originated from one of the two unrelated donors. This observation indicates the validity of matching by leukocyte typing. As a second point I would like to mention that sometimes rejection crises of the kidney can be completely overcome in the absence of treatment.

Dr. STARZL: I appreciate very much hearing about the clinical case you mentioned. Concerning your second point, I think that one would not often see spontaneous reversability of rejection crises if it were not for the basic therapy with immunosuppressants. Recently, we did orthotopic liver transplantations in about 100 dogs using azathioprine as the sole method of therapy, and we found that one half of the animals went through a crisis in which serum bilirubin values went up and then came down spontaneously without the addition of further therapy. It would seem that rejection of transplanted organs is characterized by spontaneous remissions and exacerbations.

Dr. Witz: Dr. Starzl, I wonder whether immunosuppression is the only possible mechanism for the effects of antilymphoid antisera on kidney transplantation. Is it possible that a phenomenon similar to passive immunological enhancement, as described by Kaliss, might occur in your system?

Dr. STARZL: I do not know the mechanism of the antilymphoid serum. Lymphopenia was not seen in a dog bearing a long-living liver graft. In some experiments antilymphoid globulin was given deliberately at such low levels that it had no lymphopenic effect, and yet definite therapeutic results were obtained. In most animals treated with the serum, the host spleen and lymph nodes were actually hyperplastic. Therefore the notion that the antilymphoid serum must cause lymphopenia and lymphoid atrophy to be effective requires revision.

Dr. Amos: The same sort of paradox is represented by the lack of correlation between lymphopenia and drug-induced immunosuppression.

Dr. Holland: Dr. Starzl, renal donation is not without hazard to the donor. Has any technique of genetic matchings been helpful in improving cadaver kidney usefulness?

Dr. STARzL: A dialysis center could be established at which a large number of patients could be typed, who could then be matched if a cadaveric donor became available. This is just a converse of what most centers are doing at present in which a recipient is matched against a panel of donors. There are patients who have a great number of white cell antigens 
and who are comparable, in the context of blood transfusion, to patients of AB blood type. They are "universal tissue recipients." We have done several cadaveric transplants starting 6 to 12 months ago into patients who are "universal recipients." Of these, only one has died.

DR. Regelson: In view of the fact that lymphohyperplasia occurs despite the immunosuppressive treatments applied, have agents which produce lymphocytosis, for example, pertussis vaccine or thyrotropic hormone or thyroid hormones been tried? These could be tried in association with immunosuppressive drugs in attempts to see whether the lymphoid cells are still potentially functional or whether an increased immunosuppressive effect can be obtained.

DR. STARZL: It has been suggested by Dr. Medawar that the mechanism of antilymphoid globulin may be similar to that of phytohemagglutinin, and that what the globulin does is to send the lymphoid tissue off "on a wild goose chase" with a lot of activity and no specificity.

DR. TURK: Have you seen any signs of serum sickness in patients treated with horse antiserum? Did any of these patients develop eosinophilia?

Dr. STARZL: Some of the patients developed eosinophilia. We studied quantitatively the response of patients to horse proteins by determining the antihorse protein precipitin titers which rose from $1: 2$ to $1: 16$, which was the highest level seen. On a few occasions the antibody titer returned to normal levels during treatment. Hemagglutinin titers against sheep red cells rose to levels as high as $1: 512$ in patients who had no other sign of toxicity. The clinical toxicity seen included a local reaction at the site of serum injection which tended to be much more severe on the first than on subsequent injections. Out of twelve patients, one developed hives once, another became acutely hypertensive for about 30 minutes, and a third one became hypotensive for about 10 or 15 minutes and developed air hunger. All these patients have subsequently received globulin on a number of occasions without recurrence of these effects.

DR. TURK: The rise of antibody against sheep red cells observed by Dr. Starzl in patients treated with horse antisera might be due to Forssman antigen which may be present in horse globulin as well as on sheep cells.

Dr. Milgrom: Some explanation may be offered for the graft disease that Dr. Starzl described, namely, the lesions within the grafted, nonrejected kidney. I think that immunosuppressive drugs save the graft from rejection but do not suppress significantly the formation of humoral antibodies. These humoral antibodies, directed against transplantation antigens, cannot be detected in the circulation since they are removed by the kidney. This was quite clearly demonstrated in dogs in which the humoral antibody was completely removed within hours after they were regrafted with the kidney from the original donor. Also, in humans we could not observe humoral antibodies as long as the graft was present, but we detected them after surgical removal of the graft. It seems quite plausible that the humoral antibodies directed against transplantation antigens act as Masugi's antibody in combining with antigen within the grafted kidney and inducing pathologic changes. Another point I would like to make is in connection with the use of heteroimmune sera which contain antibodies against human antigens. As Dr. Starzl demonstrated, these antibodies are not lymphocyte specific. They are just directed against man, as a species, and they combine with antigens on the cell surface. This type of antibody, from what is known, is a rather dangerous tool. I am far from criticizing Dr. Starzl's attempts, because without having anything better, he seems to be justified in trying this tool. I wonder whether this antilymphocytic serum does anything besides causing a depletion of lymphocytes. One could achieve a depletion of cannulation of lymphatics without running into the risks deriving from the use of sera which may be quite toxic for the recipient.

DR. STARZL: I do not think one could achieve with lymphocyte depletion the same effect as that seen with the antilymphoid serum. I believe that the remarks you made on another 
occasion deserve repeating, namely, that if the antiserum has any degree of specificity at all it may be not because of immunologic specificity but because the lymphocyte represents a spectacular target. In another connection I would solicit Dr. Milgrom's comments about the humoral antibodies directed against the graft which have been found in almost all our patients after renal homotransplantation.

Dr. Milgrom: I am aware of Dr. Starzl's attempts to find humoral antibodies which are primarily being detected by using sheep erythrocytes in antiglobulin consumption techniques. Most likely, differences in procedures account for different experimental results. 